\title{
CALIDAD EDUCATIVA: "UNA VISIÓN Y PRÁCTICA DE INNOVACIÓN Y DESARROLLO CURRICULAR EN EDUCACIÓN SUPERIOR PARA CENTROAMÉRICA".
}

\section{EDUCATIONAL QUALITY: "A VISION AND AN INNOVATION AND CURRICULUM DEVELOPMENT PRACTICE IN HIGHER EDUCATION FOR CENTRAL AMERICA".}

Maritza Ruiz de Campos | Post Doctora en Educación con Énfasis en Gestión Administrativa de la Educación Superior Investigadora | Universidad de Oriente

\section{Resumen}

El desarrollo del artículo hace una referencia cronológica del estado de las teorías de la educación y como éstas se han ido desarrollando través de la práctica didáctica pedagógica y como siguen predominado en algunos enfoques educativos, se aborda la educación y su relación con la gestión educativa a nivel de educación superior, las principales tendencias de la esencia e innovación curricular y de la educación, su impacto en el desarrollo para las Educación Superior en Centroamérica, la innovación y desarrollo curricular como una práctica de tecnología educativa.

La calidad educativa, debe actualmente centrarse por supuesto en una visión y práctica de innovación y desarrollo curricular a nivel de una educación superior más inclusiva, lo cual deriva de un modelo de gestión y desarrollo institucional de las instituciones para impartir los diferentes programas y carreras en el nivel de educación superior, esto conllevará a generar ranking en calidad educativa $y$, por supuesto, esto impactará como indicador de desarrollo para cada uno de las carreras y programas educativos, que lleven la excelencia como práctica en la formación de los estudiantes, y puedan también insertarse a los programas de internacionalización de la educación.

Palabras clave: Calidad educativa, desarrollo educativo, educación superior, innovación curricular, modelo educativo, práctica docente.

\section{Abstract}

This article is a chronological review of the state of different theories of education and how these have been developing throughout the teaching and pedagogical practice, and the prevalence of some educational approaches. Education and its relationship with educational 
management in higher education, their impact for the development of Higher Education in Central America, innovation and curriculum development as a practice of educational technology, are addressed.

Educational quality must be centered in a vision and an innovation and curriculum development practice, looking for a more inclusive higher education, which derives from a management model and an institutional development for teaching the different programs and degrees in the higher education level. This will promote the creation of educational quality rankings as development indicators for each and every of the programs offered, pretending to be implemented in the internationalization programs of education as well.

Keywords: Educational quality, educational development, higher education, curriculum innovation, educational model, teaching practice.

\section{Introducción}

La educación históricamente, en el mundo, es considerada como la herramienta más poderosa que transforma personas, sociedades, modelos de desarrollo, entre otros; pero pocos son los países, las instituciones y los educandos que la utilizan como parte de la transcendencia y la gestión del desarrollo. Bajo este sentido, es importante revisar dicho contexto desde el enfoque de la Gestión de la Educación Superior como una connotación epistemológica, de visión y práctica, siendo así como el presente artículo que aquí se presenta se refiere a la manera de cómo lograr una construcción de la Calidad Educativa: "Una Visión y Práctica de Innovación y Desarrollo Curricular en Educación Superior para las instituciones de educación superior de Centroamérica ", el cual aborda el enunciado como parte de una problemática actual ¿En qué sentido, el desarrollo de la educación se explica desde la construcción de la calidad educativa y la innovación del diseño curricular; y cómo los diferentes estados cronológicos explican la importancia de la educación en el desarrollo y trasformación de las humanidades, desde la práctica, visión e innovación educativa en el nivel superior para Centroamérica?. La educación se encuentra como una construcción en parámetros o tiempos que marcan grandes avances en la ciencia, la tecnología, en la investigación y extensión universitaria; y por supuesto, en las humanidades y las instituciones. El artículo, también brinda una revisión de las teorías y la filosofía de la educación desde una perspectiva epistémica y holística, analiza el desarrollo de la Educación Superior como una construcción para el desarrollo educativo y la transformación de las humanidades; que actualmente la filosofía y teoría se aloja en los modelos educativos, el desarrollo curricular, la 
calidad educativa y la gestión de la educación. Encontrándose como perspectiva de análisis, que hay una diferencia entre la historia de la educación y la pedagogía; y el posicionamiento exitoso de las instituciones que cuentan con centros de desarrollo curricular que se basan en la investigación pedagógica para trasformar o innovar el currículo, la pedagogía ha sido y sigue siendo fundamental en los procesos educativo y por su valioso aporte que ha dado al acto educativo. Por lo que se realiza un breve recorrido por las diferentes épocas y etapas por las que ha transcurrió la educación.

La pedagogía hace su aparición en la escena educativa en el momento de refinar técnicas y métodos para transmitir un conocimiento, así como teorizar sobre los hechos educativos que se presentan en cada momento histórico, esto ha impactado en la investigación, innovación y desarrollo educativo del mundo, por ejemplo; y, en especial, en Centroamérica que es importante trabajar el desarrollo curricular e innovación y desarrollo para la construcción de los modelos educativos de las Universidades. La historia de la educación va de la mano con la evolución del ser humano, no existe ninguna sociedad por primitiva que sea en la que no se presente la educación como aporte, transformación y desarrollo. Comenzando por la transferencia de simples saberes conocidos a las nuevas generaciones para su perpetuación continua, hasta el establecimiento de hábitos y costumbres, desembocando en culturas y transformadas en sociedades simples o complejas. En las culturas y sociedades, no se presentan únicamente tradiciones y sincretismos, sino que todo esto se convierte en una gama de concepciones religiosas, filosóficas y tecnológicas, que son la base de las idiosincrasias de cada país o nación. Todo esto, se fusiona en la concepción pedagógica actual y. por lo tanto, es lo que da vida y sentido de pertenencia al acto educativo que no puede verse separado de como la historia demuestra que la educación transforma, desarrolla, revoluciona, innova, entre otros; esto es un desafío actual en la Educación Superior en Centroamérica, de cómo lograr construir un modelo educativo que transforme conocimientos, hábitos, culturas, habilidades, tecnologías en los estudiantes para un mejor futuro o países diferentes.

Centroamérica, se caracteriza tipológicamente como un espacio geográfico con fuertes déficit en la economía, la educación, el desarrollo tecnológico y la innovación educativa puesto que se ve reflejado en los ranking de producción intelectual y áreas de formación , 
sin embargo, actualmente la Educación Superior tiene indicadores derivados desde las teorías educativas y modelos educativos que permiten realizar una gestión por la docencia, investigación y proyección social, vistos como componentes en materia de desarrollo educativo; pero poco se ve reflejado en ellas el componente de la formación de pedagogos o educadores con una fuerte convicción de gestión e innovación del currículo, la calidad educativa, la educación superior entendida ésta como una herramienta gestora de aprendizaje- comunidad- aula- ciencia y tecnología a nivel local, nacional y global, desde esta perspectiva análisis que se encontrará en las páginas sucesivas del presente artículo.

\section{Contexto histórico y global de la educación}

La historia de la educación, ha sido un tema de mucho debate en el mundo, y de poco interés para regiones como Centroamérica, este sentido, se define la educación en una secuencia sistemática en que la persona se desarrolla de forma histórica, la cual adquiere conocimientos, habilidades, valores, destrezas y actitudes, de tal manera que hay una transformación en el ser humano, que le sirve para toda la vida.

La educación ha evolucionado de tal manera que ha sido y es reconocida como un hecho social y por esta razón dentro de la taxonomía científica se ha ubicado en las Ciencias Sociales, porque también le permite al hombre reconocer los fenómenos como una acción, como un sistema y como un proceso en el cual todo ser humano se desarrolla, y es que se habla de la acción educativa, donde se toman en cuenta aspectos como la relación de la educación con hechos históricos, sociedad de la información ahora. Actualmente existen muchos precursores psicopedagogos que le aportaron a corrientes educativas explicadas algunas con mucho rigor epistemológico, filosófico, psicológico y educativo, que indudablemente siguen siendo corrientes utilizadas no sólo por educadores, sino que sus aportes aún han llevado a construir corrientes y enfoques hacia los modelos educativos de instituciones o centros de nivel superior, también a planes y programas de estudio; a las políticas públicas de los países, los cuales se interrelacionan, tal como se representa en el siguiente esquema: 


\section{Relación entre modelo educativo, plan educativo de país y política pública.}

Modelos Educativos: Herramientas que contemplan las bases, fundamentos y enfoques de cómo formarán a los estudiantes.
Plan Educativo de País: Herramienta de planificación, con una visión de país, que contempla programas y proyectos para la educación de un país o una nación, en plazos determinados.
Políticas Públicas: Herramienta que contiene la estrategia y bases sólidas de cómo descentralizar un programa educativo para un país o una nación.

Todas las herramientas educativas, se fundamentan en teorías y han aportado desde épocas históricas, Basadas en las hipótesis de los precursores psicoeducativos.

Fuente: Elaboración propia

Así, en Centroamérica por ejemplo, han existido diversas teorías y enfoques de como conducir los procesos de enseñanza y aprendizaje, y el desarrollo de la educación de los países, donde los aportes de pedagogos, psicólogos, filósofos, sociólogos, entre otros, han predominado en los procesos curriculares de hoy en día, ya que pasar de una formación por objetivos a una formación por competencias, pareciera una realidad fácil o dialéctica, sin embargo, siguen predominando algunas corrientes psicopedagógicas que han impactado en la forma de generar el aprendizaje, así se tienen precursores históricos que sus aporten siguen siendo válidos en algunas teorías educativas, entre ellos están Paulo Freire, Lev Vygotsky, Celestin Freinet ,Ovide Declor, Jean Jacques Rousseau, Jean Piaget, entre otros.

Por lo que entrar a la discusión e importancia de las teorías clásicas de la educación es hablar de una concepción de la educación, en general, y también de una reflexión epistémica y pedagógica muy profunda, dado que los precursores fueron ampliamente epistémicos para la construcción de los aportes psicoeducativos, muchos de ellos siguen siendo válidos para la construcción de modelos educativos o enfoques de las políticas públicas en materia educativa, que su significado tiene una connotación epistemológica, sociológica, psicológica, y aun la educación es transformadora en los ámbitos económicos de una sociedad y el mundo, históricamente también se ha destacado la educación dentro de una concepción artística y antropológica que se ha realizado una exposición poética con una reflexión profunda como lo hicieron autores holísticos como Goethe, Herder, Fichte, entre otros; esto ocurrió entre los años 1770 a 1830 por ejemplo, época en que la filosofía, de la literatura y de la pedagogía se reconocen como periodo marcado por relaciones de la 
ilustración tardía, el idealismo filosófico y pedagógico, aparece el clasicismo Alemán y las diversas corrientes del romanticismo que le apostaron a la educación de ese entonces y Centroamérica no aparece, como lo establece (Klafki, 20 I I). En este sentido, los conceptos de educación han sido de debate en plenarios con profundos análisis donde hoy en día hablar de educación también es hablar de contextos tecnológicos e innovadores, siendo así como al transcurrir los años la educación ha evolucionado, cambiado de pasar de las aulas magnas, los pódium, las pizarras de tiza, los rota folios, los acetatos a instrumentos como la computadora, el teléfono, el internet entre otros, y esto implica que la educación es la única llave transformadora de las personas y la sociedad, y también para el desarrollo de las instituciones, comunidades, regiones, países, continentes y el mundo entero, y en este caso de interés para Centroamérica.

Las teorías de la educación también se han innovado, llegando a conjeturar en la actualidad, también hacer referencia a las transformaciones de la educación y la sociedad del conocimiento, y ello es hacer referencia a las Tecnologías de la Información y Comunicación (TICs); y en Centroamérica, por ejemplo, se habla de Nuevas Tecnologías Aplicadas a la Educación, en países como Costa Rica, Panamá, El Salvador, Guatemala por ejemplo, se encuentran ya centros educativos y programas educativo virtuales ( modalidades sami presenciales, y en línea), donde los procesos han cambiado, los paradigmas de los clásicos autores y profundos constructores de análisis de la educación, la didáctica o la pedagogía han sido cuestionados en la transición de la educación, esta transformación, a este tiempo para muchos expertos los aportes de los clásico autores como Piaget siguen siendo útiles en el quehacer del aula y, sobre todo, en el acto educativo del profesor, ya que los modelos educativos de algunas universidades además de estar fundamentados de acuerdo a los abordajes de los precursores, se fundamentan en una base tecnológica e innovadora que también les permite su interrelación, para ofrecer una educación o formación integral e innovadora a través de un currículo para la formación del ser humano en ciencias o disciplinas, con el desafío de una formación de calidad, excelencia y equidad.

Al revisar, el desarrollo de la educación desde el contexto histórico y global en los países de Centroamérica, se encuentra que los planes educativos de los países, tienen su fundamento en las corrientes filosóficas de los precursores de las teorías de la educación, y que han evolucionado, sin embargo, la debilidad en las políticas públicas ha conllevado a que los planes educativos sean débiles, carentes de innovación, que poco o nada transforman, por tanto el desarrollo actual y el espíritu de transformar desde la educación es poco evidente en la práctica educativa real, es aquí donde las Universidades juegan un papel muy importante en la 
transformación de personas y territorios.

Los países de Centroamérica , actualmente están haciendo un esfuerzo como sociedades para entrar a un nuevo enfoque educativo como es la Gestión del Conocimiento y la Sociedad de la Información y en especial se expande ahora las nominadas TICs, los modelos educativos y los enfoques educativos por competencias, presuponen entonces una apertura a innovar una serie de procedimientos, recursos, gestión de programas y carreras certificadas, estrategias y herramientas que no habían sido esquemas propios de la educación tradicional, pero que con la entrada de nuevos modelos educativos implica una total renovación, por ejemplo, en los diseño de recursos didácticos, telemáticos y multimedia, esto implica una serie de cambios y una orientación hacia la educación de seres humanos más integrales, sobre todo, desde perspectivas concretas, por ejemplo, haciendo referencia cronológica y a líneas de tiempos y avances en materia educativa, se encuentran líneas de tiempo donde hubieron cambios como:

\section{De los años 1970- 2000:}

»Cambios en las concepciones: La escuela nueva donde el docente jugaba un papel de mentor, su carisma incidía en los estudiantes, considerándose esto en su tiempo como muy bueno dado que el papel del docente era comprometido y profundo en la enseñanza.

„Cambios en los recursos básicos: Gestión basada en la calidad de las profesiones, el pódium del docente era sagrado, las aulas magnas eran recursos de enseñanza, la práctica social que hacia el estudiante era incansable.

" Uso de recursos didácticos: El docente se dedicaba a preparar clases magistrales, donde era una práctica para el desarrollo de la atención en el estudiante, elaboraba papelógrafos, láminas de proyector.

»Cambios en las prácticas de los docentes y de los estudiantes: Los profesores supervisaban de forma permanente el dominio teórico y las prácticas de los estudiantes en la comunidad.

\section{De los años 2000 a la actualidad:}

" Cambios en las concepciones: Como funciona el docente en el aula, definición de procesos didácticos, identidad e innovación del docente.

"Cambios en los recursos educativos básicos: Gestión, plataformas tecnológicas, currículo y contenidos, materiales, infraestructura, acceso a redes, elaboración de materiales educativos innovadores y tecnológicos, formación del profesorado.

» Uso de recursos tecnológicos: Manipulables por el docente, por el estudiante a través de 
dispositivos móviles, campus virtuales, aplicaciones móviles, como computadoras, teléfonos, el avance del internet, tablets,

"Cambios en las prácticas de los docentes y de los estudiantes: Innovaciones curriculares, planificaciones docentes, investigación, ciencia y tecnología, innovación; ambientes de simulación, método de proyectos, inteligencias múltiples y aprendizaje integral.

"Cambios en los enfoque y modelos educativos: Muchas instituciones pasan a una escala de currículo de la formación por objetivos a la formación por competencia, rompimiento de paradigmas como la planificación didáctica, forma y entrega del proceso de enseñanza y aprendizaje, las prácticas y simulaciones del estudiante, la manera en que se evalúa, la tutoría y mediación pedagógica, fomento del emprendedurismo, enseñanza centrada en proyectos, habilidades blandas, certificación de programas y carreras, la integración de las competencias multidisciplinares e interdisciplinares, la internacionalización de la educación, la educación inclusiva y la atención a la diversidad.

En este sentido, la educación demuestra en la historia que ya no tiene solamente una connotación pedagógica, psicológica, sociológica, artística o antropológica, sino tecnológica e innovadora que rompe paradigmas didácticos y pedagógicos; donde se recogen también de manera integral algunos elementos que no se deben dejar de lado, por una parte, las concepciones sobre el proceso de la educación y su relación con la enseñanza aprendizaje, el cual es necesario ajustar para poder adaptarse a esta nueva forma de funcionamiento con respecto a la visión y esquemas preconcebidos sobre lo que es enseñar, generar aprendizaje y cómo éstos deben desarrollarse para que funcionen de acuerdo a aprendizajes preconcebidos.

Es importante considerar también en el proceso los cambios en los recursos, las TICs, los que se ponen a la disposición en las universidades, los docentes y estudiantes las más variadas formas y estrategias de aprendizaje $y$, por lo tanto, una infraestructura que responda, por supuesto, a esta política de formación educativa de equiparamiento, asesoría, capacitación, utilización y distribución de recursos al alcance de todos los protagonistas y agentes de aprendizaje, es importante señalar el replanteamiento de cara al enfoque innovador de la educación donde no solo va cambiando las gestión administrativa de la educación o la gestión académica de las Universidades, sino también la dinámica del aula y el papel que tanto estudiantes como docentes desempeñan ante nuevos retos y desafíos, donde el estudiante deja de ser un rol pasivo esperando que construyan conocimientos en su pensamiento para la vida, sino más bien autodidacta y, por supuesto, lo más importante de un 
docente es que tiene que estar a la vanguardia de los desafíos de la ciencia y la tecnología, integrando adecuadamente recursos tecnológicos e innovadores a la curricula educativa para un aprovechamiento eficiente desde sus respectivos contextos de aprendizaje.

Estas nuevas formas de innovación en el aula, requieren, por lo tanto, acciones concretas, una gestión curricular de la educación superior más integral y comprometida ,se recalca que en la medida que se atiendan los aspectos tangibles en el currículo como las plataformas de e-learning, comunicación, materiales, funcionamiento de red; y lo intangible como la comunicación pedagógica, rol, formación y actualización del profesor, interacción, diseño de actividades, proceso de evaluación y la satisfacción de estudiantes, profesores y gestores, se tendrá capacidad de construir una alternativa más cercana que la educación presencial, a distancia u online tiene sus diferencias no solo en el abordaje del currículo, sino también en la planificación y la enseñanza, si bien al principio el foco era la innovación técnica para crear entornos de aprendizaje, ahora el foco es el estudiante mismo, la metodología y los recursos del profesor o tutor, implican cambios que los gestores de la educación superior deben afrontar con planes operativos, estratégicos y desarrollo que conduzcan a contar con un currículo internacionalizado que garantice la calidad educativa de los estudiantes para que puedan enfrentar de manera responsable el mundo emprendedor, empleador o productivo al nivel local, nacional y global.

Por lo tanto, no sólo se trata de simples respuestas mecánicas y de buena disposición a emprender este proceso, sino más bien a un compromiso que pasa no solo por la adquisición de herramientas tecnológicas e innovadoras sino de una nueva gestión, preparación y una verdadera formación no sólo para docentes, sino de gestores que responda, por supuesto, a las innovaciones que como instituciones de educación afrontan.

\section{La educación y su relación con la gestión educativa universitaria.}

La educación ha tenido diversas corrientes pedagógicas, y avances tecnológicos, visto desde un contexto global, los cuales han impactado en los procesos educativos, es decir, que la educación como proceso implica "Modelos conceptuales del proceso educativo, proceso de diseño o planificación de acción educativa“ como lo establece (Gallardo, 2013).Los procesos educativos también son procesos metodológicos de intervención directos e indirectos en el acto educativo del profesor, estos procesos son conocimientos, procedimientos, valores, normas, aptitudes, hábitos, actitudes, estilos cognitivos y metacognitivos de autocontrol o autónomos, o muchas veces procesos de gestión, ya que las instituciones de educación superior cuentan con modelos para gestionar lo académico, lo administrativo y lo financiero 
para poder implementar y mantener los diferentes programas educativos, por ejemplo:

\section{Modelo de gestión en educación superior en el contexto Centroamericano.}

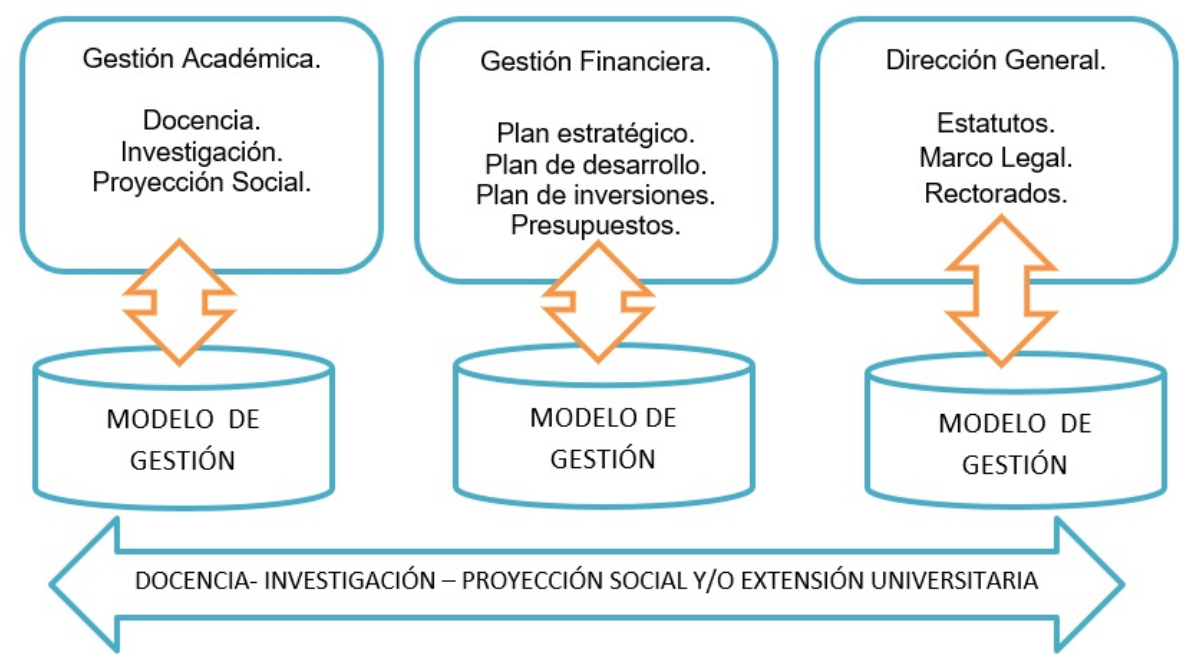

Fuente: Elaboración propia

Como es notorio en el diagrama anterior, las facultades, escuelas, los departamentos o programas educativos derivan de modelos de gestión que le apuestan a la transformación de modelos educativos interrelacionados con la docencia, la investigación, y por supuesto la extensión universitaria para formar verdaderos profesionales comprometidos con el desarrollo de sus naciones, los modelos de gestión también tienen estados de innovación que se traducen siempre en el quehacer del aula de las universidades, donde los estudiantes muchas veces construyen o no construyen actitudes y habilidades de transformación, es aquí el reto de la educación superior por ocupar ranking o niveles de calidad académica, al notar estas situaciones en los estudiantes crean programas integrales que lleven como objeto acompañar el modelo educativo construido a las propias culturas o necesidades de los estudiantes del mismo país, pero el reto es ahora el estudiantes debe interactuar con más estudiantes de diversas culturas, razas, niveles económicos, entre otros.

Sin embargo, algunos países como Costa Rica y Panamá, históricamente han sido protagonistas en el diseño de modelos educativos propios; $y$ ya hay avances en materia de educación y especialmente en la educación preescolar y media, también ya existen a nivel superior, avances sobre la calidad educativa, por ejemplo, hay acreditadoras de programas e instituciones de educación superior, donde se evalúan aspectos como: 
"A nivel de Gestión Académica: Destacan aspectos como programas y carreras, investigación, innovación tecnológica, ciencia y tecnología, producción académica y propiedad intelectual, recursos educativos y bibliotecarios, extensión universitaria, entre otros.

"A nivel de Gestión Financiera: Presupuestos, la cooperación internacional, inversiones, donaciones, becas, cuotas estudiantiles, entre otros.

"A Nivel de Dirección General: La misión, el marco legal, los reglamentos, la planeación estratégica y desarrollo institucional.

La gestión de la universidad, como lo contempla (Blanco, 2016); dependerá de su estructura organizacional y estratégica, la visión y compromiso por la educación de un país, y este compromiso lo poseen los gestores de la calidad educativa, por ejemplo; la institucionalidad universitaria; mientras el modelo de funcionamiento y desarrollo transforme la educación transcenderá el sujeto. De tal manera, que el modelo de gestión de educación superior es clave para impulsar programas y proyectos educativos encaminados hacia la formación de hombres y mujeres integrales, para insertarse a una sociedad competitiva y global, esto dependerá de las fortalezas del modelo educativo pertinente, inclusivo e innovador.

\section{Principales tendencias de la esencia curricular y de la educación, su impacto en el desarrollo de centroamérica.}

Para comprender la importancia de la gestión, es imprescindible destacar las tendencias educativas, históricamente se nota una connotación en base a los precursores que históricamente han destacado el movimiento holístico y lineal en el mundo. La gestión universitaria, ahora está referida no solo a proveer de infraestructura, de equipo docente y recursos educacionales como las aulas, sino de incluir en los programas y carrera nuevas tendencias curriculares innovadoras, inclusivas y hacer énfasis en elementos como: La educación por competencias, el currículo flexible, el aprendizaje basado en problemas y casos, la formación en la práctica, las tutorías, el currículo centrado en el aprendizaje del estudiante, el desarrollo del coaching educativo, la mediación pedagógica, el liderazgo educativo, la educación para todos, desde el enfoque de la psicología individual, como lo establece (Pérez, 2010), vistas desde una connotación de innovación y desarrollo en los recursos de aprendizaje, en primer lugar es imprescindible delimitar algunos términos básicos de esta apreciación, entre ellos se encuentra integrar currículo, la cual se entiende en este apartado como "Formar las partes en un todo, consiste en la unión deliberada de conocimiento, destrezas, actitudes y valores de diferentes áreas temáticas con el fin de 
desarrollar conocimientos y aprendizajes a través de programas o carreras, agregar elementos del aprendizaje para completar un todo, como los destaca (Escudero, 20I2).

En ese sentido, también la función básica de las TIC es ser utilizadas e integrarlas al currículo, que ha de agregarse armónicamente al currículo educativo, es decir, a un proyecto educativo, que se le agregan todas las herramientas tecnológicas y de la información, las estrategias didácticas para optimizar el proceso de enseñanza aprendizaje, esta es otra de las tendencias, entre otros. Con respecto al currículo se encuentran una serie de definiciones aparte de la ya desarrollada al inicio, que se utilizarán en particular y que recogen elementos muy completos de lo que es un currículo educativo de forma más completa e integral. En primer lugar, se encuentra que currículo es diseñar un modelo educativo capaz de comunicar los principios esenciales de una propuesta educativa, de tal forma que quede abierta al escrutinio crítico y puede ser traducida efectivamente a la práctica para formar personas de acuerdo a unos objetivos o competencias determinadas.

Las tendencias de la educación a nivel histórico, fueron bases teóricas y filosóficas que siguen siendo muchos de su principios válidos para el diseño de modelos educativos, sin embargo, en la manera de entender y concebir el hombre el mismo concepto de educación se ha innovado llegando hasta ahora a desarrollar aplicaciones de plataformas y móviles para la educación y formación de miles de jóvenes y adultos que, muchas veces, no tiene la oportunidad o posibilidad de asistir a una universidad, se hace aquí referencia a la educación virtual, lo cual, por ejemplo, se rompe aún más el paradigma no sólo de los enfoques de modelo educativos o la evaluación, sino de cómo llevar el aprendizaje o el programa educativo o de formación al estudiante, los programas de educación virtual nacieron en países desarrollados como Estados Unidos, sin embargo, ahora se ha expandido para Centroamérica, específicamente en países como Guatemala, Costa Rica, Panamá y El Salvador, tienen avances en las ofertas educativas de programas de pregrado y postgrado en línea o semi-presenciales, los cuales son sentados en los componentes de la calidad educativa siempre y cuando las carreras o programas estén sentados en modelos educativo con fundamento pedagógico, didáctico y, por supuesto, desde un contexto filosófico y teórico de la educación, que garantice una formación académica, profesional o continúa sentada en las bases $o$ indicadores de la calidad educativa acordes a las necesidades del estudiante y la sociedad, para que sean dichos programas pertinentes, seguirán siendo válidos, además del derecho que le dan los tratados internacionales de educación a las mismas personas, los países y universidades de crear espacios de educación en línea con el fin de volver la educación más inclusiva y una educación sin fronteras. 
De igual modo, una definición de currículo engloba una serie de características que no se deben pasar en el momento de definir el término currículo, se refiere "Al conjunto de competencias básicas, objetivos, contenidos, criterios metodológicos y de evaluación que los estudiantes deben alcanzar en un determinado nivel educativo". De modo general, el currículo innovador responde a las preguntas ¿Qué enseñar?, ¿Cómo enseñar?, ¿Cuándo enseñar?, ¿Dónde evaluar? , ¿Qué, Cómo y Cuándo evaluar? El currículo, en el sentido educativo, es el diseño que permite planificar las actividades académicas y educativas de una institución con la responsabilidad de la formación de hombres y mujeres en las distintas disciplinas, ciencias o áreas del conocimiento, para que puedan emplearse en diferentes instituciones, empresas, organizaciones, comunidades, sectores, industria $\circ$ aun en programas emprendedores. Es decir, que mediante la construcción curricular la institución plasma su concepción de educación para la formación ya sea para programas del nivel de pregrado o postgrado. De esta manera, el currículo permite la previsión de las cosas que ha de hacer para posibilitar la formación de los estudiantes. El concepto de currículo en la actualidad ya no se refiere sólo a la estructura formal de los planes y programas de estudio; sino a todo aquello que está en juego tanto en el aula como en la comunidad, la universidad o el centro de formación, bien como lo señala (Área, 1991), establece que el currículo educativo tiene una serie de implicaciones, entre las ideas que se pueden extraer se mencionan:

»Contiene base filosófica, pedagógica, didáctica.

»Contiene un perfil de ingreso y egreso.

"Define una serie de objetivos, competencias o saberes a obtener en el proceso de enseñanza y aprendizaje (PEA).

»Delimita el marco metodológico a implementar.

„ Fundamenta los criterios y enfoques que sustentan su propuesta.

"Orienta las acciones a seguir y su aplicación respectiva en el día a día en la universidad en los diferentes dominios taxonómicos y educativos.

"Implica todos los recursos y herramientas a utilizar como auxiliares para el proceso de enseñanza aprendizaje.

" Define y determina roles de actores del proceso de enseñanza y de aprendizaje.

»Determina los centros de práctica y desarrollo de sus aprendizajes y/o competencias.

»Contiene una reglamentación institucional y legal.

En ese sentido, lo anterior es una tarea de los departamentos de gestión y desarrollo académico o curricular, como bien lo dice la definición última citada básicamente el currículo 
muestra en este sentido el ¿Qué, ¿Cómo, ¿Cuándo Enseñar y Qué Evaluar? . Ahora bien, la situación es que se tienen por un lado es que las TICs muchas están ausentes en el Currículo Educativo al que se quieren integrar ante necesidades de enseñanzas, es aquí donde cabe implementar un proyecto institucional de Gestión Curricular que sea capaz de incorporar los elementos del currículo y las herramientas o recursos tecnológicos a la práctica didáctica que realiza el docente en el aula, pero siempre basado en teoría o filosofía educativa y acorde al modelo educativo que, en principio, logre transformar al estudiante, para que éste transforme su comunidad o país.

Por lo tanto, la integración del currículo a la práctica educativa, tiene implicaciones de responsabilidad y uso de estrategia didáctica como es la utilización de todos los recursos y herramientas que le facilitan las TICs con el fin de enriquecer el proceso de enseñanza. La integración curricular, es una práctica innovadora, la información curricular implica el uso de teorías y tecnologías para lograr un propósito en el aprender de un concepto, un proceso, en una disciplina curricular específica o una ciencia. Se trata de valorar las posibilidades didácticas de las TICs en relación con objetivos y fines educativos a la innovación curricular de ahora. Al integrarlas curricularmente se pone énfasis en el aprender y cómo éstas pueden apoyar aquello, sin perder de vista que el centro es el aula y es el aprender para la vida.

En este mismo sentido, La Sociedad Internacional de Tecnología en Educación (ISTE) define la ICT como una efectiva integración de las TICs, que se logra cuando los estudiantes son capaces de seleccionar herramientas tecnológicas para obtener información en forma actualizada, analizarla, sintetizarla y presentarla profesionalmente. La tecnología debería llegar a ser parte integral de cómo funciona la clase y tan accesible como otras herramientas utilizadas en la clase.

Bien lo señala (Escudero, 1989), que en la actualidad el gran problema no es la carencia de recursos e información como en otras épocas, el problema es la sobreabundancia de información y herramientas tecnológicas, y el desafío es como utilizarla de la mejor manera en los procesos de enseñanza con un uso didáctico, y como sumarlas o integrarlas al currículo. Siendo así como, la institución se convierte en reguladora, que le permite orientar y capacitar al docente para educar a los estudiantes analizar y hacer procesos mediante los cuales se filtre toda la información para que su proceso de enseñanza sea más significativo y no solo para su educación académica sino aquella que le servirá el resto de su vida en los diferentes contextos que se podrá movilizar y que de alguna forma le será de utilidad en su campo laboral y la vida. La integración y pertenecía es en tanto un proceso que permite que las herramientas se usen en todo el proceso didáctico y pedagógico, sin perder de vista que el 
fin último es el proceso de enseñanza y aprendizaje (PEA) con las facilidades que ofrecen las TICs por sí mismas, y como auxiliares que permitan aprovechar todo el potencial que contienen para optimizar el aprendizaje. Por tanto "La integración e innovación curricular también implica":

»Identificar programas y carreras de formación pertinentes al contexto.

"Diseñar enfoques educativos integrales, con un enfoque innovador y de forma transparentemente usando las tecnologías y los recursos dentro del diseño de currículo.

»Utilizar las tecnologías para planificar estrategias y facilitar la construcción del aprender.

"Aplicar las tecnologías por parte de docente, tutores o estudiantes de forma responsable y atinente a los contenidos o saberes.

»Orientar las tecnologías para apoyar las clases, las prácticas y simulaciones.

"Utilizar las tecnologías como parte del diseño y contenido del currículo.

» Usar las tecnologías para aprender el contenido de una disciplina o una ciencia.

» Diseñar software educativo para la enseñanza de una disciplina o ciencia, con responsabilidad.

En este proceso, la integración no consiste únicamente en traer una serie de enfoques, herramientas y recursos tecnológicos a una carrera e introducirlo al aula y al proceso de enseñanza aprendizaje, y que son suficientes por sí mismos, sino más bien cómo hacerlo de forma provechosa e innovadora, que trasforme en el estudiante y éste al país o la sociedad, para el caso es lo que se requiere para los países de Centroamérica, un currículo pertinente.

No es por lo tanto suficiente, que se tenga un equipo técnico innovador, una infraestructura que cumpla con todos los estándares y/o poseer software para los diferentes niveles de lo más adaptado e innovador posible, si no se cuenta con un plan estratégico curricular integral que responda a la filosofía institucional y al currículo de la institución, un equipo docente que esté capacitado para el manejo y para utilizarlo en el proceso de enseñanza y por supuesto un estudiante que goce de las condiciones propicias para su uso y aplicación , se convertirá en un profesional que recibe o recibió un proceso de aprendizaje que se convierte en el conocimiento o las competencias de su carrera para insertarse como un profesional altamente responsable a la sociedad o el país .

Como ya se ha venido manejando la integración de las TICs, al currículo es otra manera innovadora de diseño curricular de hoy en día, es una transformación para el docente actual y no se reduce a un hecho mecánico meramente en el cual se tengan equipos avanzados como el software, sino a todas las herramientas que parten esencialmente de una visión curricular, 
de una formación y capacitación docente, de una formación al estudiante con nuevas visiones de enseñanza adaptadas a las circunstancias actuales de enseñanza, sin perder de vista los modelos curriculares con sus dominios didácticos y pedagógicos. Es importante, enseñar a los estudiantes a usar el equipo y las herramientas con fines educativos y provechosos, ellos vienen de una sociedad saturada de tecnología y de información, se necesita que en este proceso aprendan cómo usarla con fines educativos y utilidad significativa, como el aprendizaje puede ser diferente, enriquecedor $y$, por supuesto, adaptado a los mecanismos metacognitivos para una mejor aprehensión del conocimiento y hacer más holístico el proceso de aprendizaje, tomar en cuenta algunos requerimientos para la integración curricular, en las últimas tendencias es importante tomar en cuenta :

"Una filosofía educativa de partida que valore sus posibilidades didácticas en el proceso educativo en el marco de los objetivos de la universidad e insertadas en el proyecto educativo. La integración curricular de las TICs, pasa por una política y estrategia de implementación. Esta nace de una filosofía de gestión en donde se deben tener claros los alcances y por supuesto los beneficios, así como los costos que implicaría la implementación de dicha tecnología y recursos.

"Asumir un cambio de rol del docente y del estudiante: El rol del docente ya no volverá a ser el mismo. El docente ahora es un facilitador mediante el uso de los medios y procedimientos metodológicos que como auxiliares funcionan las TICs y por supuesto, el estudiante deja de ser un receptor pasivo de información sino más bien se convierte en un agente autodidacta u autónomo que gestiona su propio aprendizaje y utiliza los medios a la par del docente, facilitándose de esa forma la adquisición de los contenidos o las competencias a desarrollar en el proceso, el profesor debe ser creativo al momento de palmear la clase o la práctica con recursos tecnológicos.

»Que el Currículo oriente el uso de las TICs: En este sentido, se ha mencionado que vienen a complementar el proceso de enseñanza aprendizaje, es decir, sirven como auxiliares para optimizar y mejorar e interactuar este proceso, que esten al servicio del currículo, las tecnologías por sí misma no es suficiente sino hay una adecuada implementación didactica y utilidad con fines de formación y aprendizaje que derive desde la práctica del docente.

» La gestión de los centros de investigación e innovación curricular, o de ciencia y tecnología, diseñen y cuentes con planes de equipamiento de aulas, software, tutores de e-learning, simuladores educativos, laboratorios, centros de práctica con recursos tecnológicos, que permitan a los docentes y estudiantes contar con accesibilidad en los diferentes momentos del aprendizaje.

Los nuevos desafíos tecnológicos, de innovación tecnológica y curricular, y científicos, 
orientan nuevas estrategias de gestión institucional y de enseñanza esto, por lo tanto, implica reorientar el currículo de tal forma aprovechar todos los recursos que posibilitan estos medios y contar con un currículo innovador global internacionalizado. Las Tecnologías de la información y la comunicación son un instrumento y su finalidad es, que el estudiante no sea pasivo en las tecnologías por sí mismas, sino más bien activo en el acto o proceso de enseñanza aprendizaje que es el fin último de dicha integración.

Por lo tanto, la concreción de un proyecto curricular innovador es una estrategia educativa que actualmente reta la Educación Superior en Centroamérica, requiere de lanzarse a la innovación curricular con el fin de brindar desde el modelo educativo un aprendizaje que no sólo sea dinámico e interactivo sino también permita que sea lo más autodidacta posible. Donde el docente se motive y comprenda que los estudiantes, de hoy en día, aprenden diferente a hace veinte años, por ejemplo; también es necesario enfatizar hacia los estudiantes la disciplina y esfuerzo que le permitan hacer una correlación de sus aprendizajes donde integre adecuadamente todas las tecnologías de información y comunicación en los diferentes procesos educativos a fin de aprovechar dichos recursos $y$, por supuesto, los proceso a parte de individuales pueden también ser grupales, por lo que las habilidades en el uso requeridas y desarrolladas deben estar directamente relacionadas con el contenido y las tareas de la clase.

La aplicación curricular no responden a un uso arbitrario e improvisado de las diferentes disciplinas o ciencias, sino más bien a un proceso sistemático y, por lo tanto, estructurado de tal forma que el proceso de enseñanza resulte más adecuado, pertinente y factible para el estudiante, que es lo que se busca como fin último, es decir, que en materia educativa no sólo basta con que se hable de integrar un modelo educativo al currículo, sino más bien de que se adapte también a un enfoque educativo que transforme al estudiante para la vida y este lleve una visión de desarrollo que pondrá en práctica como profesional, es decir que se encamina hacia una nueva tendencia que puede emprenderse desde los centros de ciencia y tecnología, innovación y desarrollo o líneas de investigación de los centros de desarrollo pedagógico, y en esta medida los programas de formación impactaran en las competencias de los estudiantes.

\section{La innovación y desarrollo curricular como práctica en la educación superior para una mejor Centroamérica.}

Las nuevas tendencias curriculares, generan impacto a través de las teorías de la educación y los modelos educativos, derivan un currículo innovador, y la práctica ha demostrado que han sido y siguen siendo de mucha importancia en los países como Costa Rica, Panamá, entre 
otros; países clasificados en vías de desarrollo dentro del ámbito centroamericano, donde cada Institución de Educación Superior en el marco de la organización y gestión interna, diseña y pone en marcha lineamientos claros sobre la gestión y la actividad curricular, docente e investigadora de los profesores desde un sistema de gestión y de calidad educativa basada en los principios de la innovación tecnología o curricular, en aras de dar respuestas a sus aspiraciones, necesidades y requerimientos en materia académica y laboral; y propicios para el desarrollo académico institucional, donde se propicia que el profesorado además de sus horas de docencia, desarrollen durante su tiempo la actividad investigadora o de extensión.

Asimismo frente a las oportunidades que ofrece la revolución de las tecnologías de la información tienen la oportunidad de dinamizar el modelo educativo desde una práctica innovadora y pertinente, más emprendedora y de flexibilidad curricular en busca de la movilidad estudiantil $y$ docente, y que tienen consciencia que hay necesidad de fortalecer los rendimientos académicos de docentes y estudiantes, aquí es necesario que a nivel de educación superior se implementen programas de formación sobre la actividad docenteinvestigadora, para desarrollar las competencias necesarias que permitan a los investigadores desenvolverse técnica y científicamente en este campo para generar conocimiento y contribuir al desarrollo científico y tecnológico. Acerca de la dedicación a la docencia y a la investigación, se encuentra que las tendencias internacionales presentan los rasgos centrales como la calidad educativa, la gestión académica, la investigación, la innovación, la producción intelectual, la internacionalización y la estandarización de programas académicos a nivel de pregrado y postgrado como nuevas tendencias para las Universidades de Centroamérica.

Los diferentes países buscan conciliar entre la dedicación a la docencia, la investigación o innovación, existiendo diferencias según diversos aspectos de los académicos e instituciones en que se desempeñan, para el caso de los países de Centroamérica los rankings de producción académica en baja. A nivel general, los académicos se han volcado tanto hacia la docencia y poco a la investigación e innovación, donde el diseño curricular es otra de las nuevas tendencias y prácticas de innovación en educación superior.

En Centroamérica, la docencia ha superado a la investigación, la dedicación de los académicos debe enfocarse a la docencia, investigación e innovación como el futuro de la educación superior, y esto se debe a la gestión institucional que ha predominado y es un reto para los directores de investigación apostarle a la dinamización de la investigación a nivel interno y externo, pero para ello se requiere apoyo de los gestores institucionales y de 
planes de desarrollo con un compromiso y visión de desarrollo estratégico. El desarrollo de la educación superior muchas veces tiene complejidades de tipo social, político, económico, cultural, entre otros, que trascienden las capacidades institucionales; $y$ el compromiso de los docentes universitarios, esto requiere de la incidencia de otras instancias y actores de la política gubernamental, de los colegios de profesionales, de las asociaciones, del sector productivo, entre otros; para impulsarlo y trascender fronteras en el campo académico se requiere de una gestión institucional integral en Red, lo cual es una tarea y compromiso que sumen los actores responsables dedicados a la Educación Superior para una mejor Centroamérica.

La internacionalización de la educación superior, es uno de los retos en la práctica de la innovación curricular, para enfatizar en el currículo innovador la internacionalización en un sentido muy positivo y coherente con los valores académicos que corresponde al fortalecimiento del carácter universal del aprendizaje, se requiere de los conocimientos y de la investigación, el establecimiento de redes, a la cooperación solidaria y a la facilitación de la movilidad y gestión, que puede impactar en el desarrollo profesional del profesorado y de los gestores administrativos, para la mejora de la calidad de los aprendizajes de los estudiantes, abriéndole espacios y construyendo escenarios para ingresar a procesos de acreditación institucional y certificación internacional ya sea de programas, laboratorios u otros recursos educacionales que garanticen la calidad educativa, la cual juega un rol importante para promover un cambio de rumbo en educación superior, por ejemplo, en materia de gestión las prácticas y resultados reconocidos a nivel nacional e internacional, contribuyen al desarrollo endógeno y exógeno de un país, a formar un nuevo tejido social profesional para las personas y el país, es aquí cuando se habla de impacto de un currículo, es porque un currículo se fundamenta en una teoría y en una filosofía educativa que a cualquier institución le garantizará transcender hacia la calidad educativa.

Es necesario abrir oportunidades de mejora en las áreas del ejercicio docente, apoyo a la investigación e innovación, a los centros de investigación pedagógica, en educación superior. No pueden haber procesos y resultados exitosos en el desarrollo de la educación superior si se continúan utilizando, sin fundamento filosófico y teórico los enfoques educativos, estrategias de innovación que surgieron y funcionaron para estudiantes de hace más de un siglo, los estudiantes de hoy en día están altamente influenciados por las tecnologías de la información y la comunicación, que pertenecen a la era digital, implicando esto innovar el currículo hacia esas nuevas formas de como los estudiantes aprenden y que pueden superar 
las habilidades tecnológicas de un docente.

Es necesaria la actualización curricular, desarrollar consciencia del valor de los aprendizajes a lo largo de la vida, para responder con garantías de éxito a la formación de los nuevos modelos educativos y los profesionales en la sociedad del conocimiento, identificados como nativos digitales, pero todo ello sentado en un diseño de currículo con fundamento filosófico teórico y didáctico pedagógico. En forma sistemática se han alfabetizados digitalmente muchos docentes a través de planes o programas de profesionalización; y han logrado tener un dominio fuerte en el manejo instrumental de las herramientas tecnológicas para la práctica educativa, que contribuye a formar hombres y mujeres como personas y como profesionales integrales.

Fortalecerles el dominio científico e innovador de su disciplina de estudios, sus niveles de comprensión lectora, la capacidad para convivir armónicamente con sus semejantes, el interés y aprovechamiento en la lectura, el sentido de responsabilidad en sus compromisos académicos, la práctica de principios y valores ciudadanos, resolver problemas de la vida cotidiana, afrontar con honradez los dilemas éticos de su profesión, mantener el equilibrio socioemocional frente a situaciones imprevistas, aprender científicamente, capacidad para administrar, localizar, interpretar y reflexionar sobre información requerida y su contenido, entre otros, la forma de lograr los objetivos de aprendizaje o las competencias, debe derivarse del diseño curricular del modelo educativo innovador; $y$ esto derivando una práctica docente más atinada a las necesidades de los estudiantes, al desarrollar su actividad laboral será con un enfoque humanista y socialmente comprometido, aspectos que las máquinas por si solas, jamás podrán hacer; lo que vuelve el quehacer docente como una actividad insustituible en los procesos de enseñanza y aprendizaje, frente a los avances logrados artificial, mecánica y tecnológica, todo esto no se lograra fuera de un enfoque filosófico de la educación.

La adaptación a los cambios en la incertidumbre actual, en pro del desarrollo de la educación y la construcción de una nueva ciudadanía, requiere enfrentar desafíos y uno de ellos es el encausarse como reto en el rumbo determinado por el protagonismo que cobra el aprendizaje sobre la enseñanza. El reto de transitar del ejercicio de una docencia basada en la enseñanza, la recepción de conocimientos y el saber enciclopédico a otra basada en el aprendizaje y desarrollo de competencias que gira en torno al estudiante, en el cual los planteamientos didácticos adoptados por diversos países de Centroamérica en el marco de la 
Declaración de Bolonia por ejemplo, se hacen acompañar de una serie de trasformaciones en las estructuras de la educación superior que deben ser del conocimiento, comprensión e interpretación por parte de los docentes, en aras que las interrelacionarlos con el quehacer académico y que incentiven su motivación, identificándose con los esfuerzos orientados a la mejora de la calidad educativa, todo ello implica contar con un currículo innovador pertinente e inclusivo como lo señala la Organización de las Naciones Unidas para la Educación, la Ciencia y la Cultura (UNESCO).

Muchas Universidades de Centroamérica, cuentan con plantas de docentes muy experimentados con fuertes competencias para la docencia, la investigación e innovación tecnológica, , ya sea por la sólida formación académica y años de trabajo en su especialidad disciplinar, así como por la experiencia adquirida por el paso de los años, lo cual puede ser aprovechado para la inducción y formación de los nuevos cuadros docentes o profesores nóveles talentos, sean a tiempo parcial o asociados, que constituyen, potencialmente, relevos generacionales para las plantas docentes de tiempo completo que pueden apostarle a la implementación de un currículo innovador capaz de transcender y transformas personas y sociedades, sin embargo muchas veces no se valoran estos académicos institucionalmente.

El ejercicio docente y académico en la Educación Superior, requiere de un saber y capacidades profesionales amplias, el cual históricamente se ha señalado que, tradicionalmente, el docente de educación superior desarrolla su saber profesional y aprende a enseñar por descubrimiento durante el quehacer laboral cotidiano o por la asesoría que le pueden brindar los colegas más experimentados. Al respeto se ha considerado que el aprendizaje profesional para guiar adecuadamente los procesos de aprendizaje, se desarrolla, ante todo, en el día a día de unas comunidades vivas de práctica, orientadas por profesorados comprometidos con la educación. Un buen ejercicio docente, debe tener inmerso un saber filosófico, pedagógico, didáctico, metodológico y hasta andragógico que derive de un modelo educativo, que contribuya a la comprensión del docente universitario como profesional de la educación; los sentidos y alcances del concepto de saber pedagógico y la importancia de la práctica docente como fuente de conocimiento pedagógico y de mejoramiento de las prácticas educativas sentadas en bases filosóficas educativas deriva un currículo innovador.

En el marco de su autonomía institucional y a través de sus sistemas de gestión de calidad, en la educación superior se incluyan e implementen procesos de desarrollo profesional integrando capacitación, acompañamiento, diálogo, mediación pedagógica fundamentada en la psicología individual, sistematización de experiencias, formación postgradual, investigación, 
desarrollo, innovación y reflexión sobre la propia práctica, de tal manera que todo ello lleve a que desarrollen y estén bajo un papel más protagónico en materia de formación docente, ya que es una práctica muy comprometedora para las futuras generaciones, sin embargo sigue siendo un desafío ante la visión de que la Universidad va cambiando.

Es muy importante que en educación superior se cuente con cuerpos docentes y gestores formados integralmente con fuerte compromiso ético, que estén muy atentos a las diversas y aceleradas dinámicas de cambio que surgen en el devenir de la sociedad y que le exigen una conexión más cercana con los problemas y la presentación de alternativas de solución para los mismos, las instituciones formadoras a la par de su autonomía, como potestad para tomar sus propias decisiones y como expresión de confianza que la comunidad, en general, les ha depositado, deben actuar con libertad y frente a la formación integral de los profesionales, la producción, custodia, difusión y aplicación del conocimiento, la investigación, la reflexión crítica de la realidad, así como la extensión y proyección social al hacer referencia al rol de los agentes de educación superior, con sus cuerpos de profesores investigadores, se encuentran marcadas diferencias entre los diferentes países, lo que podría explicar el porqué de las diferencias en los niveles y calidad de vida de la población entre los diversos Estados, al relacionar desarrollo científico y tecnológico, con productividad y avances en los niveles académicos o educativos de los países, es aquí el reto y desafío para la la innovación y desarrollo curricular como práctica de la educación superior para Centroamérica y para la transformación de personas y países.

\section{Conclusión}

La educación tiene una connotación epistémica, que se explica desde la historia y las teorías pedagógica y filosóficas, que en el presente articulo aborda como enunciado y que explica el desarrollo de la educación, se explica desde la construcción epistémica de las teorías y la filosofía, y como los diferentes estados cronológicos explican la importancia de la educación en el desarrollo y trasformación de las humanidades y las instituciones ; la educación es considerada entonces como una herramienta transformadora, es la llave que abre puertas al desarrollo de personas, comunidades, poblaciones ,instituciones, organizaciones y aun territorios sin fronteras y límites.

La educación, es transformadora y sigue demostrando combatir la reflexión "La Educación Transforma y Educa para toda la Vida"; en Centroamérica han existido precursores pedagógicos que han logrado transformar ideales y paradigmas desde la educación, en los 
últimos años la educación superior ha transcendido desde los modelos educativos, programas y proyectos pensados endógenamente, y construidos desde la innovación curricular; y que son descentralizados desde sus departamentos de extensión universitaria o centros de investigación pedagógica con miras y en aras de la búsqueda de un desarrollo educativo más pensado y más planificado.

En ese sentido, el artículo que aquí se presenta hace un abordaje en la construcción epistémica de la educación sobre todo en los modelos de gestión universitaria, y los retos de la innovación curricular para contar con modelos educativos pertinente, lo que se pretende es dejar una reflexión macro constructiva que lleve al sujeto a reflexionar que la educación tiene un sentido místico en cuanto a la práctica pedagógica y didáctica que realiza por ejemplo el educador o instructor en el aula; es decir, que las personas que se dedican a la educación debe ver el ámbito de práctica docente como una actividad responsable y ética, y comprometido que los esfuerzos que hacen las universidades de innovación y desarrollo curricular como práctica de la educación superior. Asimismo, los gestores de las Universidades deben proponerse nuevos retos ante la Universidad que va cambiando en un mundo más global y mas inclusivo, y comprender que la educación es la llave para trascender hacia la formación de hombres y mujeres, y el desarrollo de una institución o un país.

\section{Bibliografía}

ANZALDÚA ARCE R. E. (2009). La formación: una mirada desde el sujeto", enhttp://www.comie.org.mx/ congreso/memoriaelectronica/v10/pdf/area_tematica_15/ponencias/025 I-F.pdf.

AREA, M. (I99I): Los medios, los profesores y el curriculum, $1^{\circ} \mathrm{Ed}$. Barcelona.

Blanco Hernández, Ingrid (2016). La gestión académica "Criterio Clave de la Calidad de la Gestión de las Instituciones de Educación Superior”, Universidad de Cartagena; Colombia.

ESCUDERO, J.M. (1989): La integración de las nuevas tecnologías en el curriculum y el sistema escolar. Tecnología Educativa-Nuevas tecnologías aplicadas a la educación. $1^{\circ}$ Ed. Madrid.

ESCUDERO, J.M. (20I2): La integración de las nuevas tecnologías en el curriculum y el sistema escolar. Tecnología Educativa-Nuevas tecnologías aplicadas a la educación. $1^{\circ}$ Ed. Madrid.

GALLARDO LÓPEZ, B. (2013): La Teoría de la Educación, Objetos y Enfoques, $I^{\circ}$ Ed. España.

GORDOKIN, I. C. (2005)" La formación docente y su relación con la epistemología", en Revista lberoamericana de Educación No.37/5. Revista editada por la Organización de estados Americanos (OEI).

KLAFKI, W. (20I I). La Importancia de la Teorías de la Educación para la Concepción de la Educación General e Hoy, 
Universidad de Marburgo. Alemania.

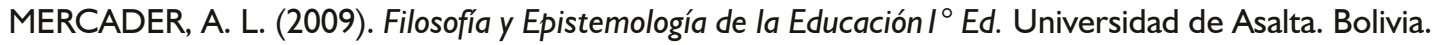

PERDOMO INTERIANO, C. R. (2009). Filosofía de la Educación. I I Ed. Pearson. México.

PÉREZ. M. (2010). Tendencias y Retos de la Innovación Curricular, I Ed. México.

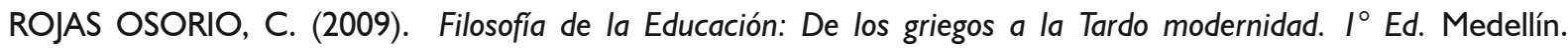
Universidad de Antioquia. Italia.

TUNNERMANN, C. (2008). La Educación Superior en América Latina y El Caribe. I Ed. UNESCO. Colombia.

TUNNERMANN, C. (2008). La Educación Superior en el Umbral del Siglo XXI. I Ed. Colección y Ediciones CRESALC. Venezuela. 